\title{
Importance of Geriatric Syndrome Screening within 48 Hours of Hospitalization for Identifying Readmission Risk: A Retrospective Study in an Acute-Care Hospital
}

\author{
Jinyoung Shin ${ }^{1,2}$, Seol-Heui Han ${ }^{2,3}$, Jaekyung Choi ${ }^{1,2}$, Yoon-Sook Kim ${ }^{2,4}$, Jongmin Lee ${ }^{2,5}$ \\ ${ }^{1}$ Department of Family Medicine, Konkuk University Medical Center, Konkuk University School of Medicine, Seoul, Korea \\ ${ }^{2}$ Research Institute on Healthy Aging, Konkuk University Medical Center, Seoul, Korea \\ ${ }^{3}$ Department of Neurology, Konkuk University Medical Center, Seoul, Korea \\ ${ }^{4}$ Department of Quality Improvement, Konkuk University Medical Center, Seoul, Korea \\ ${ }^{5}$ Department of Rehabilitation Medicine, Konkuk University Medical Center, Konkuk University School of Medicine, Seoul, Korea
}

Corresponding Author:

Jongmin Lee, $\mathrm{MD}, \mathrm{PhD}$

Department of Rehabilitation

Medicine, Konkuk University Medical

Center, 120-1 Neungdong-ro,

Gwangjin-gu, Seoul 05030, Korea

E-mail: leej@kuh.ac.kr

ORCID:

https://orcid.org/0000-0001-8718-0099

Received: March 24, 2020

Revised: April 20, 2020

Accepted: May 1, 2020
Background: Given the association between geriatric syndrome and hospital readmission, we evaluated the suitability of geriatric syndrome screening for care (GSC) in identifying readmission risk and suggested the appropriate time for GSC. Methods: GSC considering cognitive impairment, depression, polypharmacy (five or more medications), functional mobility, dysphagia, malnutrition, pain, and incontinence was performed among 2,663 general ward inpatients aged 65 years or older within 48 hours after admission and again before discharge between November 2016 and October 2017. From each patient, fall events, pressure ulcers, potentially inappropriate medication use, and delirium were assessed at admission. Patients were divided into two groups on the basis of readmission within 1 year after the first admission. According to the screening period (at admission and before discharge) and in-hospital decline, we applied receiver operating characteristic curve analysis to compare the prevalence of clinical concerns between the readmission and no-readmission groups. We also used multiple logistic regression analysis to evaluate the risk of readmission according to the presence of geriatric syndrome and clinical outcomes. Results: The 782 readmitted patients (29.4\%) showed a higher rate of poor GSC than those who were not readmitted. Polypharmacy at admission was significantly correlated with readmission risk (area under the receiver operating characteristic curve $=0.602$ ). Fall events (odds ratio $[O R]=4.36 ; 95 \%$ confidence interval $[\mathrm{Cl}], 2.36-8.05)$, urinary incontinence $(\mathrm{OR}=4.21 ; 95 \% \mathrm{Cl}$, 3.28-5.39), and depressive $\operatorname{mood}(\mathrm{OR}=3.88 ; 95 \% \mathrm{Cl}, 2.69-5.59)$ at admission were risk factors for readmission. Conclusion: Geriatric syndromes assessed by GSC at admission was associated with an increased risk of readmission.

Key Words: Patient readmission, Aged, Geriatric assessment, Risk assessment

\section{INTRODUCTION}

Readmission is an important quality and safety issue in healthcare research and is also a tremendous burden on patients and their families. ${ }^{1)}$ The cost of unplanned readmissions among Medicare patients in the United States was as high as $\$ 26$ billion in 2014. ${ }^{2)}$ The older population, for whom medical expenditures and rates of hospital readmission are higher relative to those among individuals of other age groups, is growing in Korea. ${ }^{3)}$ The risk factors for readmission have been evaluated widely and include both patient-related factors such as demographic characteristics, diagnosis, comorbidities, and healthcare utilization as well as provider and health system-related factors such as hospital location and healthcare costs. $^{3,4)}$ A risk-stratified approach has recently been proposed for 
identifying patients at high risk of readmission for transitional care interventions, which includes assessments of disease severity, hospital-acquired complications, and stability on discharge.

The Korean Framework for Senior-Friendly Hospitals ${ }^{6)}$ was developed with reference to the Taiwanese ${ }^{7)}$ and Canadian ${ }^{8)}$ systems and was first implemented to address the need for healthcare assessment tools for older Korean inpatients in our acute-care hospital in 2016. Since May 2016, geriatric syndrome screening for care (GSC) has been performed for all older inpatients aged 65 years or older both at admission and before discharge. For further diagnostic assessment, patients with clinical issues are referred to a multidisciplinary team consisting of medical doctors specializing in neurology, rehabilitation medicine, family medicine, internal medicine, and psychology; nurse practitioners; dieticians; pharmacists; and quality improvement facilitators. Further, patients may receive personalized and comprehensive consultations throughout their hospital stay based on the GSC results. All steps are logged in each patient's electronic health record (EHR) and shared with the attending medical staff.

Although readmission risk assessment throughout older patients' hospitalization is important, ${ }^{6-8)}$ continuous evaluation of patients in busy in-patient units can be cumbersome. Unfortunately, exactly when it is appropriate to assess the risk of readmission remains unclear.

Therefore, the present study aimed to identify the appropriate time for readmission risk assessment and confirmed a simple comprehensive screening approach for the assessment of readmission risk by GSC in older patients.

\section{MATERIALS AND METHODS}

\section{Study Participants}

We retrospectively enrolled 3,570 patients aged 65 years or older who were admitted to the general ward of a tertiary hospital with 800 beds located in Seoul, South Korea between November 2016 and October 2017. After excluding patients with 1-day hospitalization ( $n=668 ; 18.7 \%$ of enrolled patients), localized disease such as ophthalmological conditions $(n=163 ; 4.6 \%)$, and incomplete records on the factors evaluated $(n=76 ; 2.1 \%)$, the remaining 2,663 patients were finally examined. Study population was divided into two groups on the basis of readmission within 1 year regardless of diagnosis. The readmission group was defined as patients with more than one hospitalization during the observation period, whereas the no-readmission group was defined as patients who experienced only one hospitalization during the same period.

The Institutional Review Board of the Clinical Research Ethics Committee of Konkuk University Medical Center approved the exemption for this study and allowed the authors to review the patients' records (No. 11701347). The need for informed consent was also waived.

\section{Demographics and Comorbidities}

We obtained data on age, sex, health insurance status, body mass index (BMI), smoking status (current smoker, nonsmoker, or former smoker), alcohol intake (no alcohol intake, less than once per week, or more than once per week), duration of hospital stay, marital status (married/with a partner or divorced/without a partner), route for hospitalization (scheduled from an outpatient clinic or abruptly from an emergency visit), and reason for admission (e.g., surgery) from the EHRs. Comorbidities were defined as hypertension, diabetes mellitus, stroke, cardiovascular disease, cancer, respiratory disease, mental disorder, or other according to the attending physician's diagnosis or prescription. We counted the number of comorbidities per patient.

\section{Assessment of geriatric syndrome}

Geriatric syndrome was assessed not only within 48 hours of admission but also before discharge. An attending nurse designated at admission assessed the following nine GSC domains in each patient: cognitive impairment, ${ }^{9)}$ depressive mood, ${ }^{10)}$ polypharmacy (five or more medications), ${ }^{11)}$ functional immobility, ${ }^{12,13)}$ dysphagia, ${ }^{14)}$ malnutrition, ${ }^{15)}$ urinary incontinence, ${ }^{16)}$ fecal incontinence, ${ }^{17)}$ and pain. ${ }^{18)}$ A multidisciplinary team trained the nurses who conducted this assessment through face-to-face training and monitored all GSC results. The GSC questionnaire is shown in Supplementary Table S1. Functional mobility was categorized as "independent", "requires assistance", or "unable to perform" regarding climbing stairs, walking to a toilet, and transferring from a bed to a chair or a wheelchair. Patients categorized as "requires assistance" or "unable to perform" were considered to have functional immobility. The dysphagia screening test was performed using a simplified dysphagia symptom questionnaire. ${ }^{14)}$ Malnutrition was defined as a score of more than two for the sum of the responses to weight loss in the last 6 months (yes $=1$, no $=0$ ) and decreased appetite (yes $=1$, no $=0$ ). ${ }^{15)}$ The other screening questions, including the presence of cognitive impairment, depressive mood, polypharmacy (five or more medications), incontinence in the last month, and pain score ( $\geq 4$ points), were relevant when participants responded "yes". The attending nurses were able to complete this survey for each patient within 5 minutes since the GSC can be done simply using EHRs.

\section{Assessment of Other Clinical Outcomes}

An attending nurse also surveyed fall events, the presence of pres- 
sure ulcers, potentially inappropriate medication use, and delirium risk within 48 hours of admission. A fall event was defined as at least one fall within 90 days before the day of admission. ${ }^{9)}$ Pressure ulcers were defined as partial skin loss, deep craters in the skin, a skin break exposing muscle or bone, or necrotic ulcer. ${ }^{9)}$ Potentially inappropriate medication use was defined according to the updated 2012 American Geriatrics Society Beers Criteria. ${ }^{19)}$ On the basis of these criteria, two pharmacists categorized patients who were taking more than one medication into the inappropriate medication use group. Delirium risk was evaluated using the Nursing Delirium Screening Scale, which consists of five items: disorientation, inappropriate behavior, inappropriate communication, illusion or hallucinations, and psychomotor retardation. ${ }^{20)}$ Each domain was scored as 1 (yes) or 0 (no) point(s). We considered patients with total scores of more than 2 points to be at risk for delirium.

\section{Statistical Analysis}

Descriptive statistics were expressed as mean \pm standard deviation for continuous variables and as numbers (proportions) for categorical variables. These data were compared between the readmission and no-readmission groups using Student t-test and chisquare test. In-hospital decline was defined as any aggravation of assessment findings between admission and discharge. Patients with no change or with an improvement in these findings were categorized into the "no in-hospital decline" group. The prevalence of readmission was compared according to the time of assessment (at admission or before discharge) and in-hospital decline using chisquare test. Receiver operating characteristic (ROC) curves were used to test the ability to predict the readmission risk. An area under the ROC curve (AUC) value of 0.5 represents a variable with no discriminating ability, whereas an AUC of 1.0 represents a variable with perfect discrimination. ${ }^{21)}$

We evaluated the odds ratios (ORs) and 95\% confidence intervals (CIs) for the risk of readmission according to the presence of geriatric syndrome and other clinical outcomes using a multiple logistic regression analysis after adjusting for age, sex, BMI, marital status, smoking, alcohol intake, number of comorbidities, duration of hospital stay, reason for admission, route for hospitalization, and type of health insurance. Additionally, we performed stratified analysis according to sex and the 75th percentile of hospital stay ( $<13$ days and $\geq 13$ days). As an increased risk of readmission in female patients has been reported, ${ }^{3)}$ we confirmed that similar findings were apparent in our study. We examined the statistical significance of the modifying effects of sex and the duration of hospital stay using a generalized linear model after adjusting for confounding factors. All analyses were performed using IBM SPSS Statistics for Windows, version 24.0 (IBM Corp., Armonk, NY,
USA).

\section{RESULTS}

Among the 2,663 participants, 782 (29.4\%) were readmitted (Table 1) during the observation period. Age, sex, smoking status,

Table 1. Baseline characteristics of the study population.

\begin{tabular}{|c|c|c|c|}
\hline Variable & $\begin{array}{c}\text { No } \\
\text { readmission } \\
(\mathrm{n}=1,881)\end{array}$ & $\begin{array}{c}\text { Readmission } \\
(\mathrm{n}=782)\end{array}$ & p-value \\
\hline Age (y) & $75.1 \pm 6.9$ & $75.2 \pm 6.7$ & 0.486 \\
\hline Sex & & & 0.176 \\
\hline Male & $884(47.0)$ & $390(49.9)$ & \\
\hline Female & $997(53.0)$ & $392(50.1)$ & \\
\hline Smoking & & & 0.774 \\
\hline Current & $177(9.4)$ & $74(9.5)$ & \\
\hline Never/former & $1,704(90.6)$ & $708(90.5)$ & \\
\hline Alcohol intake & & & 0.341 \\
\hline Never/less than one time per week & $1,637(87.0)$ & $674(86.2)$ & \\
\hline More than one time per week & $244(13.0)$ & $108(13.8)$ & \\
\hline Body mass index $\left(\mathrm{kg} / \mathrm{m}^{2}\right)$ & $23.9 \pm 3.7$ & $23.7 \pm 4.0$ & 0.003 \\
\hline Hospital stay (day) & $11.2 \pm 13.9$ & $12.7 \pm 14.3$ & 0.153 \\
\hline Reason for admission & & & $<0.001$ \\
\hline Surgery & $813(43.2)$ & $224(28.6)$ & \\
\hline Others & $1,068(56.8)$ & $558(71.4)$ & \\
\hline Marital status & & & 0.966 \\
\hline Married/with partner & $1,824(97.0)$ & $741(94.8)$ & \\
\hline Unmarried/without partner & $57(3.0)$ & $41(5.2)$ & \\
\hline Comorbidity & & & 0.096 \\
\hline None & $238(12.7)$ & $97(12.4)$ & \\
\hline Hypertension & $733(39.0)$ & $289(37.0)$ & \\
\hline Diabetes mellitus & $207(11.0)$ & $85(10.9)$ & \\
\hline Stroke & $32(1.7)$ & $11(1.4)$ & \\
\hline Cardiovascular disease & $122(6.5)$ & $58(7.4)$ & \\
\hline Cancer & $68(3.6)$ & $46(5.9)$ & \\
\hline Respiratory disease & $55(2.9)$ & $35(4.5)$ & \\
\hline Mental disorder & $14(0.7)$ & $4(0.5)$ & \\
\hline Others & $412(21.9)$ & $157(20.1)$ & \\
\hline Type of health insurance & & & 0.180 \\
\hline National health insurance & $1,744(92.7)$ & $734(93.9)$ & \\
\hline Medical aid program & $137(7.3)$ & $48(6.1)$ & \\
\hline Route for hospitalization & & & 0.830 \\
\hline Scheduled from outpatient clinic & $1,233(65.6)$ & $516(66.0)$ & \\
\hline Abruptly from emergency visit & $648(34.4)$ & $266(34.0)$ & \\
\hline Fall events & $20(1.1)$ & $38(4.9)$ & $<0.001$ \\
\hline Pressure ulcer & $123(6.5)$ & $90(11.5)$ & $<0.001$ \\
\hline Inappropriate medication use $(\geq 1)$ & $129(6.9)$ & $101(12.9)$ & $<0.001$ \\
\hline Nursing delirium screening scale $(\geq 2)$ & $109(5.8)$ & $63(8.1)$ & 0.154 \\
\hline
\end{tabular}

Values are presented as mean \pm standard deviation or number (\%). 


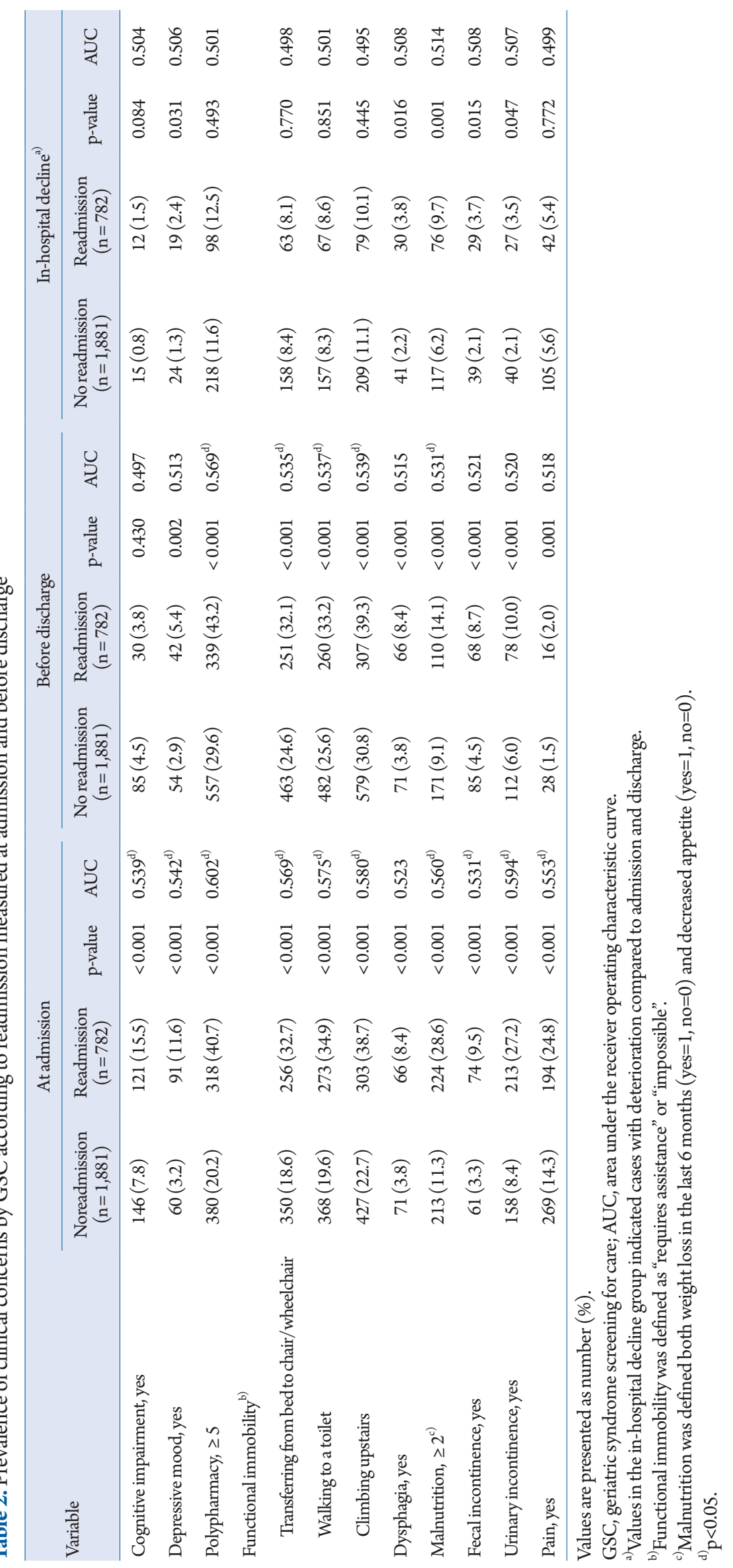


alcohol intake, marital status, comorbidities, health insurance type, route for hospitalization, and duration of hospital stay did not differ between the readmission and no-readmission groups. Patients in the readmission group showed a lower BMI value and admission rate for surgery but higher frequencies of fall events, pressure ulcers, and inappropriate medication use than those in the no-readmission group. However, there was no difference in delirium scale scores between the two groups.

Table 2 shows the prevalence of clinical concerns observed during GSC at admission, before discharge, and during in-hospital decline. The readmission group showed a higher frequency of clinical concerns by GSC at admission than that in the no-readmission group (all $\mathrm{p}<0.001$ ). However, the difference in cognitive impairment between the readmission and no-readmission groups disappeared before discharge $(p=0.430)$. We observed significant differences in the number of patients who experienced in-hospital decline relating to depressive mood, dysphagia, malnutrition, and fecal and urinary incontinence between the readmission and no-readmission groups (all $\mathrm{p}<0.05$ ). Polypharmacy, functional immobility, and the presence of pain showed similar rates of de- cline during hospitalization in both groups. Screening for geriatric syndromes at admission showed a higher AUC value than the GSC recorded before discharge or the GSC of in hospital decline group. Polypharmacy (five or more medications per day) and urinary incontinence at admission were significantly associated with readmission ( $\mathrm{AUC}=0.602$ and 0.594 , respectively).

The risks of readmission according to the presence of geriatric syndromes and other clinical outcomes assessed within 48 hours after admission are shown in Table 3. After adjusting for confounding factors, we found that all measured variables increased the risk of readmission. The ORs were highest for fall events $(\mathrm{OR}=4.36$; 95\% CI, 2.36-8.05), urinary incontinence (OR=4.21; 95\% CI, 3.28-5.39), and depressive mood ( $\mathrm{OR}=3.88$; 95\% CI, 2.69-5.59). In stratified subgroup analysis, delirium in men, and cognitive impairment, dysphagia, and delirium in patients with hospital stays of longer than 13 days did not increase the OR of readmission. Furthermore, pressure ulcers in patients with hospital stays of $<13$ days did not increase the OR of readmission, whereas a hospital stay of $\geq 13$ days did ( $\mathrm{OR}=4.89,95 \% \mathrm{CI}, 2.92-8.18$ ) ( $\mathrm{p}$-interaction $<0.001)$. Hospital stays of $<13$ days tended to be associated with

Table 3. Comparison of readmission according to the geriatric syndrome and clinical outcomes

\begin{tabular}{|c|c|c|c|c|c|}
\hline \multirow{2}{*}{ Variable } & \multirow{2}{*}{ Total $^{\mathrm{a})}$} & \multirow{2}{*}{ Male $^{\text {b) }}$} & \multirow{2}{*}{ Female $^{\mathrm{b})}$} & \multicolumn{2}{|c|}{ Hospital stay $^{c)}$} \\
\hline & & & & $\geq 13$ days & $<13$ days \\
\hline Cognitive impairment & $2.46(1.84-3.29)$ & $2.17(1.43-3.30)$ & $2.80(1.86-4.22)$ & $1.44(0.85-2.45)$ & $3.19(2.25-4.53)$ \\
\hline Depressive mood & $3.88(2.69-5.59)$ & $4.17(2.34-7.41)$ & $3.74(2.32-6.05)$ & $3.54(1.80-6.99)$ & $4.04(2.62-6.24)$ \\
\hline Polypharmacy & $2.63(2.15-3.22)$ & $3.17(2.38-4.22)$ & $2.20(1.64-2.95)$ & $2.64(1.81-3.85)$ & $2.67(2.10-3.40)$ \\
\hline \multicolumn{6}{|l|}{ Functional immobility } \\
\hline Transferring from a bed to a chair/wheelchair & $2.58(2.03-3.28)$ & $2.55(1.80-3.61)$ & $2.63(1.88-3.68)$ & $1.79(1.20-2.67)$ & $3.11(2.30-4.21)$ \\
\hline Walking to a toilet & $2.60(2.05-3.30)$ & $2.72(1.93-3.83)$ & $2.51(1.80-3.49)$ & $1.83(1.23-2.72)$ & $3.07(2.28-4.14)$ \\
\hline Climbing up stairs & $2.47(1.94-3.03)$ & $2.54(1.84-3.49)$ & $2.33(1.70-3.20)$ & $1.80(1.22-2.66)$ & $2.72(2.07-3.59)$ \\
\hline Dysphagia & $2.65(1.78-3.94)$ & $2.69(1.55-4.69)$ & $2.62(1.47-4.68)$ & $1.34(0.73-2.47)$ & $4.56(2.64-7.87)$ \\
\hline Malnutrition & $3.32(2.61-4.22)$ & $4.05(2.88-5.71)$ & $2.77(1.96-3.91)$ & $3.10(1.99-4.83)$ & $3.23(2.41-4.34)$ \\
\hline \multicolumn{6}{|l|}{ Incontinence } \\
\hline Fecal & $3.39(2.25-5.10)$ & $3.82(2.09-6.97)$ & $3.36(1.89-5.95)$ & $2.32(1.19-4.53)$ & $4.59(2.71-7.77)$ \\
\hline Urinary & $4.21(3.28-5.39)$ & $4.06(2.81-5.87)$ & $4.54(3.23-6.39)$ & $3.85(2.46-6.04)$ & $4.42(3.27-5.96)$ \\
\hline Pain & $2.06(1.64-2.60)$ & $2.21(1.58-3.09)$ & $1.98(1.44-2.73)$ & $2.08(1.37-3.14)$ & $2.07(1.56-2.73)$ \\
\hline Fall events & $4.36(2.36-8.05)$ & $4.07(1.73-9.61)$ & $4.70(1.93-11.45)$ & $6.21(2.40-16.01)$ & $3.14(1.37-7.17)$ \\
\hline Pressure ulcers ${ }^{\mathrm{d})}$ & $1.92(1.35-2.73)$ & $2.01(1.23-3.29)$ & $2.00(1.19-3.38)$ & $4.89(2.92-8.18)$ & $0.93(0.56-1.56)$ \\
\hline Inappropriate medication use, $\geq 1$ & $1.90(1.41-2.58)$ & $2.00(1.31-3.05)$ & $1.79(1.15-2.80)$ & $2.16(1.26-3.70)$ & $1.84(1.27-2.67)$ \\
\hline Nursing delirium screening scale, $\geq 2^{\mathrm{d})}$ & $1.62(1.08-2.44)$ & $1.44(0.81-2.58)$ & $1.91(1.07-3.41)$ & $1.01(0.56-1.82)$ & $2.81(1.56-5.04)$ \\
\hline
\end{tabular}

Values are presented as odds ratio (95\% confidence interval). All variables were measured within 48 hours after admission.

${ }^{\text {a) }}$ Multiple logistic regression analysis after adjusting for age, sex, body mass index (BMI), marital status, smoking, alcohol intake, number of comorbidities, duration of hospital stay, reason for admission, route for hospitalization, and type of health insurance.

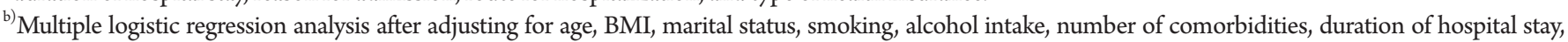
reason for admission, route for hospitalization, and type of health insurance.

${ }^{c}$ Multiple logistic regression analysis after adjusting for age, sex, BMI, marital status, smoking, alcohol intake, number of comorbidities, reason for admission, route for hospitalization, and type of health insurance. Hospital stay was divided into the 75th percentile of duration (13 days).

d) p-interaction $<0.05$ which was only analyzed according to the hospital stay. 
an increased risk of delirium in older inpatients hospitalized for acute care ( $\mathrm{p}$-interaction $<0.05)$.

\section{DISCUSSION}

In this study of Korean older inpatients, geriatric syndromes were associated with an increased risk of readmission in an acute-care hospital. The presence of geriatric syndromes assessed by GSC at admission, rather than before discharge or the occurrence of in-hospital decline, was associated with the risk of readmission. Polypharmacy at admission showed the highest discriminating ability for readmission. Among the GSC criteria and other clinical outcomes, a fall event before hospitalization was a significant risk factor for readmission among Korean older patients.

GSC was developed for administration by medical staff, caregivers, or patients in both clinical and nonclinical settings. GSC is a multidimensional and interdisciplinary screening tool encompassing medical, psychological, and functional domains. Therefore, it can be extended to transitional care settings even when the medical staff is not adequately trained or is too occupied to manage complex geriatric needs. ${ }^{18)}$ Delirium, cognitive impairment, depressive mood, and inappropriate medication use are often not recognized in older patients, a fact that underscores the need for better geriatric care. ${ }^{18)}$ Therefore, an easily administered tool is important to promptly evaluate inpatients for the presence of geriatric syndromes. In this context, our study confirmed the effectiveness of screening using GSC within 48 hours of hospitalization rather than before discharge or assessing in-hospital decline to determine the readmission risk. Although in-hospital decline and stability at discharge in older patients with pneumonia are considered good predictors of readmission risk, ${ }^{22)}$ only one study has reported this correlation; thus, it had low generalizability, in addition to other limitations such as incomplete ascertainment of readmission and a lack of validation. ${ }^{5)}$ The study was further limited by including only pneumonia patients.

Similar to the results of our study, a study in the United States found that polypharmacy (six or more medicines) at admission predicted the risk of 30-day readmission. ${ }^{23)}$ Polypharmacy was also a major risk factor for readmission in a prospective study of older Italian inpatients, with similar OR and 95\% CI values (2.72; 1.484.99) ${ }^{24)}$ While potentially inappropriate medication use did not increase the risk of readmission in the Italian study, the results of the present study confirmed an increased risk of readmission. This difference could be attributed to the fact that the previous study assessed readmission during a 6-month period in contrast to the 1 -year period in our study. ${ }^{24)}$ The previous study may not have observed an association between readmission and potentially inap- propriate medication during the short-term observation period.

The results of our study confirmed that fall events before hospitalization increased the risk of readmission. In addition, long-term hospitalization ( $>13$ days) was associated with the presence of pressure ulcers, which is a common problem in older inpatients. ${ }^{9)}$ However, relatively short-term hospitalization conferred a higher risk of delirium than did longer hospital stay, although the difference was not statistically significant. Therefore, delirium, which develops or is aggravated by acute environmental changes, requires attention during the care of older patients. ${ }^{25)}$

A systematic review of 12 studies including 3,590 patients reported that in-hospital geriatric assessment and co-management had no overall effect on readmission within 30 days $(\mathrm{OR}=1.28$; 95\% CI, 0.71-2.31; $\mathrm{n}=695)$ and within 12 months (OR=0.91; 95\% CI, 0.64-1.29; $\mathrm{n}=601$ ). Unfortunately, limited reduction in the length of hospital stay (pooled mean difference $=-1.88$ days; $95 \% \mathrm{CI},-2.44$ to -1.33 ) and in-hospital mortality (pooled $\mathrm{OR}=0.72 ; 95 \% \mathrm{CI}, 0.50-1.03$ ) after geriatric assessment and co-management were reported, with low-quality evidence. ${ }^{26)} \mathrm{Al}$ though we did not administer any interventions based on the GSC finding, our results suggest that a long hospital stay related to pressure ulcers could mediate the risk of readmission; thus, it may confer an effect modification, as shown previously. ${ }^{5,26)}$

Among GSC variables, dysphagia alone did not provide an acceptable AUC ( $p>0.05)$. Dysphagia can cause respiratory complications, including aspiration pneumonia and malnutrition. ${ }^{27)}$ One explanation for this finding is the lower prevalence of dysphagia in our participants, including those in the readmission group (8.4\%), relative to those among acute-care older inpatients in Spain $(47.4 \%)^{28)}$ and Denmark (50\%). ${ }^{29)}$

Compared to the GSC results at admission, pain, cognitive impairment, and urinary impairment improved on the assessment before discharge. Whereas pain is thought to have been controlled after hospitalization, the other factors may not be improved with short hospitalization. This change may be due to positive responses by the participants to the medical staff's questions that has been treated, resulting in a response bias in the before-discharge questionnaire, which may have reduced the relevance of the findings to readmission. ${ }^{30)}$ In contrast, the prevalence of polypharmacy, functional immobility, and fecal incontinence before discharge increased compared to that at admission, possibly due to the treatment process.

Logging all care processes in EHRs and sharing records with attending physicians play a specialized and comprehensive role in consultation in the hospital setting. ${ }^{31)}$ Furthermore, EHRs facilitate communication among medical staff and organizations and provide structured coordination and documentation of the medi- 
cal services provided. ${ }^{32)}$ Therefore, GSC performed within 48 hours of hospitalization using the EHRs of all inpatients aged 65 years or older is expected to be efficient.

Still, the present study has some limitations. First, it was conducted in a single institution; thus, the findings cannot be generalized to other hospital settings without further review. However, screening for geriatric syndromes by GSC within 48 hours can provide information on patients at high risk of readmission in similar institutions. Second, instead of assessing the exact cause of hospitalization in the current study, we adjusted for whether the hospitalization was for surgery based on the EHR. Additionally, we could not confirm whether the causes of readmission were related to previous admissions or to other hospitals, or to pre-admission residence. Since the various associations between geriatric syndromes and individual diagnoses were not significant in this study and could be confounded by detection bias, we simplified the participant-related variables. Additionally, comorbidities and medication use were assessed based on self-reports rather than medical record review, which might have caused bias toward positive answers because of social desirability. ${ }^{30)}$ Unfortunately, we could not assess all medical records of all participants as they were not shared between different medical institutions. The no-readmission group may have included patients who were admitted to other hospitals, as we did not consider visits or admission to other hospitals in this analysis.

In conclusion, geriatric syndromes assessed by GSC was associated with an increased risk of readmission. The results of our study support the importance of an integrated approach regarding readmission in older inpatients. Further research is needed to determine the efficacy of GSC related to transitional care or other hospital settings.

\section{ACKNOWLEDGMENTS}

\section{CONFLICT OF INTEREST}

The researchers claim no conflicts of interest.

\section{FUNDING}

This study was supported by Konkuk University in 2020 .

\section{AUTHOR CONTRIBUTIONS}

Conceptualization, JS, SHH, JC, YSK, JL; Data curation, SJ, JC, YSK; Investigation, JS, JC, YSK; Methodology, JS, SHH; Project administration, $\mathrm{SHH}$, JL; Supervision, $\mathrm{SHH}$; Writing original draft, JS, JL; Review and editing, SHH, JC, YSK.

\section{Supplementary Materials}

Supplementary materials can be found via https://doi.org/10.4235/ agmr.20.0017.

\section{REFERENCES}

1. Goldstein JN, Hicks LS, Kolm P, Weintraub WS, Elliott DJ. Is the care transitions measure associated with readmission risk? Analysis from a single academic center. J Gen Intern Med 2016;31: 732-8.

2. Boozary AS, Manchin J 3rd, Wicker RF. The Medicare hospital readmissions reduction program: time for reform. JAMA 2015; 314:347-8.

3. Robinson S, Howie-Esquivel J, Vlahov D. Readmission risk factors after hospital discharge among the elderly. Popul Health Manag 2012;15:338-51.

4. Wang YC, Chou MY, Liang CK, Peng LN, Chen LK, Loh CH. Post-acute care as a key component in a healthcare system for older adults. Ann Geriatr Med Res 2019;23:54-62.

5. Weinreich M, Nguyen OK, Wang D, Mayo H, Mortensen EM, Halm EA, et al. Predicting the risk of readmission in pneumonia: a systematic review of model performance. Ann Am Thorac Soc 2016;13:1607-14.

6. Kim YS, Lee J, Moon Y, Kim HJ, Shin J, Park JM, et al. Development of a senior-specific, citizen-oriented healthcare service system in South Korea based on the Canadian 48/6 model of care. BMC Geriatr 2020;20:32.

7. Chiou ST, Chen LK. Towards age-friendly hospitals and health services. Arch Gerontol Geriatr 2009;49 Suppl 2:S3-6.

8. Wong KS, Ryan DP, Liu BA. A system-wide analysis using a senior-friendly hospital framework identifies current practices and opportunities for improvement in the care of hospitalized older adults. J Am Geriatr Soc 2014;62:2163-70.

9. Lakhan P, Jones M, Wilson A, Courtney M, Hirdes J, Gray LC. A prospective cohort study of geriatric syndromes among older medical patients admitted to acute care hospitals. J Am Geriatr Soc 2011;59:2001-8.

10. Lachs MS, Feinstein AR, Cooney LM Jr, Drickamer MA, Marottoli RA, Pannill FC, et al. A simple procedure for general screening for functional disability in elderly patients. Ann Intern Med 1990;112:699-706.

11. Gnjidic D, Hilmer SN, Blyth FM, Naganathan V, Waite L, Seibel MJ, et al. Polypharmacy cutoff and outcomes: five or more medicines were used to identify community-dwelling older men at risk of different adverse outcomes. J Clin Epidemiol 2012;65: 
989-95.

12. Tinetti ME. Performance-oriented assessment of mobility problems in elderly patients. J Am Geriatr Soc 1986;34:119-26.

13. Lee Y, Lee HH, Uhm KE, Jung HJ, Kim YS, Shin J, et al. Early Identification of Risk Factors for Mobility Decline Among Hospitalized Older Patients. Am J Phys Med Rehabil 2019;98:699705.

14. Uhm KE, Kim M, Lee YM, Kim BR, Kim YS, Choi J, et al. The Easy Dysphagia Symptom Questionnaire (EDSQ): a new dysphagia screening questionnaire for the older adults. Eur Geriatr Med 2019;10:47-52.

15. Ferguson M, Capra S, Bauer J, Banks M. Development of a valid and reliable malnutrition screening tool for adult acute hospital patients. Nutrition 1999;15:458-64.

16. Abrams P, Cardozo L, Fall M, Griffiths D, Rosier P, Ulmsten U, et al. The standardisation of terminology in lower urinary tract function: report from the standardisation sub-committee of the International Continence Society. Urology 2003;61:37-49.

17.Elsawy B, Higgins KE. The geriatric assessment. Am Fam Physician 2011;83:48-56.

18. Deschodt M, Van Grootven B, Jeuris A, Devriendt E, Dierckx de Casterle B, Dubois C, et al. Geriatric CO-mAnagement for Cardiology patients in the Hospital (G-COACH): study protocol of a prospective before-after effectiveness-implementation study. BMJ Open 2018;8:e023593.

19. American Geriatrics Society 2012 Beers Criteria Update Expert Panel. American Geriatrics Society updated Beers Criteria for potentially inappropriate medication use in older adults. J Am Geriatr Soc 2012;60:616-31.

20. Gaudreau JD, Gagnon P, Harel F, Tremblay A, Roy MA. Fast, systematic, and continuous delirium assessment in hospitalized patients: the nursing delirium screening scale. J Pain Symptom Manage 2005;29:368-75.

21. Hoo ZH, Candlish J, Teare D. What is an ROC curve? Emerg Med J 2017;34:357-9.

22. Capelastegui A, Espana Yandiola PP, Quintana JM, Bilbao A, Diez R, Pascual S, et al. Predictors of short-term rehospitaliza- tion following discharge of patients hospitalized with community-acquired pneumonia. Chest 2009;136:1079-85.

23. Logue E, Smucker W, Regan C. Admission data predict high hospital readmission risk. J Am Board Fam Med 2016;29:50-9.

24. Fabbietti P, Di Stefano G, Moresi R, Cassetta L, Di Rosa M, Fimognari F, Bambara V, Ruotolo G, Castagna A, Ruberto C, Lattanzio F, Corsonello A. Impact of potentially inappropriate medications and polypharmacy on 3-month readmission among older patients discharged from acute care hospital: a prospective study. Aging Clin Exp Res 2018;30:977-84.

25. Setters B, Solberg LM. Delirium. Prim Care 2017;44:541-59.

26. Van Grootven B, Flamaing J, Dierckx de Casterle B, Dubois C, Fagard K, Herregods MC, et al. Effectiveness of in-hospital geriatric co-management: a systematic review and meta-analysis. Age Ageing 2017;46:903-10.

27. Martin A, Ortega O, Roca M, Arus M, Clave P. Effect of a minimal-massive intervention in hospitalized older patients with oropharyngeal dysphagia: a proof of concept study. J Nutr Health Aging 2018;22:739-47.

28. Carrion S, Cabre M, Monteis R, Roca M, Palomera E, Serra-Prat $\mathrm{M}$, et al. Oropharyngeal dysphagia is a prevalent risk factor for malnutrition in a cohort of older patients admitted with an acute disease to a general hospital. Clin Nutr 2015;34:436-42.

29. Melgaard D, Rodrigo-Domingo M, Morch MM. The prevalence of oropharyngeal dysphagia in acute geriatric patients. Geriatrics (Basel) 2018;3:15.

30. Paulhus DL. Measurement and control of response bias. In: Robinson JP, Shaver PR, Wrightsman LS, editors. Measures of personality and social psychological attitudes. St. Louis, MO: Academic Press; 1991.p. 17-59.

31. Shin J, Ko H, Kim JA, Song YM, An JS, Nam SJ, et al. Hospital cancer pain management by electronic health record-based automatic screening. Am J Manag Care 2018;24:e338-e343.

32. Tomoaia-Cotisel A, Farrell TW, Solberg LI, Berry CA, Calman NS, Cronholm PF, et al. Implementation of care management: an analysis of recent AHRQ research. Med Care Res Rev 2018; 75:46-65 